\title{
Optical fiber sensors of magnetic field applying Faraday's effect
}

\author{
Tadeusz Pustelny, Cuma Tyszkiewicz, Kamil Barczak \\ Silesian University of Technology, Institute of Physics, \\ ul. Bolesława Krzywoustego 2, 44-100 Gliwice, Poland, e-mail: pustelny@zeus.polsl.gliwice.pl.
}

\begin{abstract}
This work presents a system for measuring magnetic field intensity. The idea of measurements is based on the influence of an external magnetic field on the polarization state of single-mode light propagated along optical fibers. In the paper, some experimental results of test investigations for the measurements of the magnetic field of high intensity are presented. The ultimate aim of the investigation outlined below is to work out magnetic field intensity sensors which, together with optical fiber temperature sensors and electric field intensity sensors, will make it possible, in the nearest future, to entirely monitor the operation of electromagnetic power units such as, e.g., high voltage power transformers.
\end{abstract}

Keywords: optical fiber sensors, magnetooptic effects, state of polarization.

\section{Introduction}

The optical fiber magnetic field sensors are an interesting alternative for classical ones since they possess good insulating properties and can use optical fibers as electromagnetic-insensitive feeding conduits. However, this choice also has some disadvantages. The main problem with this type of sensors is their high susceptibility to mechanical vibrations [1]. A satisfactory solution to that problem will allow us to put easily into effect the project of using the optical fiber magnetic field sensors for various practical applications [2].

This paper deals with an investigation of Faraday's effect in a few quartz fibers. The results obtained enable production of magnetic field sensors based on optical fibers. The optical fibers applied to the measuring set-up, which employs Faraday's effect, are used not only as good light wave conduits but also as sensing components.

The Optoelectronic Department at the Silesian University of Technology has been conducting investigations to design and elaborate optical fiber sensors for various applications for several years. 


\section{Theoretical remarks}

Magnetic field can influence light propagation by changing the material properties which are described by the dielectric constants. This influence modifies real or imaginary parts of the constants, thus changing the refraction and absorption indices. In the case of Faraday's effect the magnetic field changes the real part of the dielectric constant tensor triggering off the appearance of circular birefringence [2]-[4]. For optically isotropic materials without birefringence and eigenabsorption, two waves with opposite circular polarisation propagating in the material are observed as a result of Faraday's effect [3]. There is a difference between refraction indices $\Delta n=n^{\prime}-n^{\prime \prime}$ of both waves which, in turn, results in some difference of phases $\Delta \psi$ between the waves after passing a distance $l$. This phase difference is equal to [3]

$$
\Delta \psi=2 \pi \frac{\left(n^{\prime}-n^{\prime \prime}\right) l}{\lambda}
$$

where $\lambda$ is the value of wavelength of the light wave in vacuum.

As a result of their interference the linear polarization is twisted by a certain angle with respect to the input polarisation. The twisting angle is equal to [3]

$$
\Gamma=\frac{1}{2} \Delta \psi=\frac{\pi l\left(n^{\prime}-n^{\prime \prime}\right)}{\lambda} .
$$

Based upon the theory of molecular optics [4], [5], Faraday's twisting angle can be given as follows:

$$
\Gamma=V H l \cos \Theta
$$

where $V$ - Verdet material constant [rad/A], $H$ - magnetic field intensity, $\Theta$ - an angle between the light wave vector and the magnetic field intensity vector.

Equation (3) shows that Faraday's effect reaches its maximum when the angle between the magnetic field intensity vector $\mathbf{H}$ and the light wave vector $\mathbf{k}$ is equal to zero $\left(\Theta=0^{\circ}\right)$. Since both vectors are reciprocally parallel, the effect is named a longitudinal one [5].

The important property of Faraday's effect is non-reversibility. It consists in the absence of twisting reduction when the light propagation changes its direction through $180^{\circ}$ (e.g., after reflection). After reversing the direction of light propagation the rotation of the polarization plane will be reverse. However, the twisting angle (which has arisen before the light direction change) will be still increasing. It should be underlined that the non-reversibility property of Faraday's effect will make it possible to multiply easily this effect and to achieve proper sensitivity [6].

\section{Measuring position}

Figure 1 shows the set-up of the system for magnetic field measurements by usiltg Faraday's effect in optical fibers. A light ray of wavelength $670 \mathrm{~nm}$ is emitted by an 


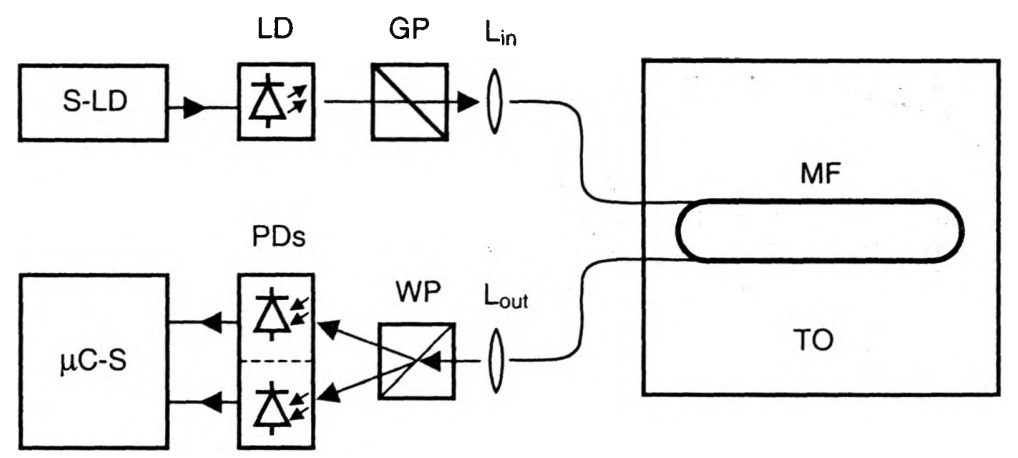

Fig. 1. System for magnetic field measurement: S-LD - laser diode supply, LD - laser diode, GP - Glan's polarizer, WP - Wollaston's polarizer, $\mathrm{PDs}$ - photodetectors, $\mathrm{L}_{\text {in }}, \mathrm{L}_{\text {out }}$ - lenses, $\mu \mathrm{C}-\mathrm{S}$ - microcomputer with measuring card, MF - magnetic-field sensor, TO - air-core testing coil.

laser diode. The ray enters into a fiber after passing by the Glan's polarizer (GP). The magnetosensing part of the fiber is wound round on a special reel and put into the testing magnetic field [6].

At the testing stage, an air-core coil (TO) is used as a magnetic field source in the measuring system. The coil has a rectangular hole to place inside it a special reel with the fiber being tested. The coil is supplied with direct current ranging from 0 to $20 \mathrm{~A}$. For current intensity of $20 \mathrm{~A}$ the average magnetic field intensity being calculated on the reel surface is about $H \approx 10^{5} \mathrm{~A} / \mathrm{m}$.

The second end of the fiber is provided with selfoc type lens in order to collimate a light ray. The ray after passing the selfoc comes across Wollaston's prism (WP) and is split into two waves, which have mutually orthogonal polarization planes. These waves are detected by the photodetectors (PD) which measure the intensity of light in each ray. Next, voltages of the PDs are measured with a PC card (Adantech, PCI-1711 type) which is demanded by the system and mounted in the computer. The system works in Lab-View environment and the measurement processes are fully automated. Such an arrangement allows us to determine the parameters which characterize the polarization state ellipse of the wave leaving the fiber before its investigation.

\section{Method of polarization state detection}

The aim of polarization analysis is to define the degree of polarization, its type and parameters that define the state of polarization [1], [5], [6]. There are three possible types of polarization: linear, circular and elliptical. In the case of linear polarization only the azimuth must be determined and in the case of circular polarization only the helicity must be determined. As regards elliptical polarization one must determine the azimuth, angle of ellipticity and helicity.

The authors have elaborated a new method of determining the state of polarization. The method has been presented in detail in [6]. In this paper, the method is used for determination of the azimuth $\alpha$ and the modulus of an ellipticity angle $|v|$. Measured 


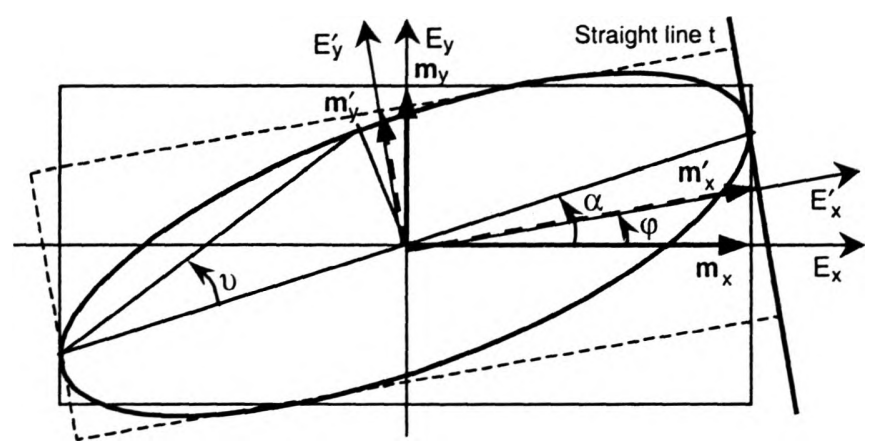

Fig. 2. Determination of the ellipse of the polarization state.

by PDs the values of voltage $U_{\mathrm{PD} 1}$ and $U_{\mathrm{PD} 2}$ are appropriately equal to parameters $m_{x}^{2}$ and $m_{y}^{2}$ of the ellipse of polarization, as shown in Fig. 2 .

In order to determine the state of polarization the measurement of $U_{\mathrm{PD} 1}$ and $U_{\mathrm{PD} 2}$ should be performed twice. The first measurement should be done when the axes of a polarizer overlap the axes of co-ordinate system $E_{x}, E_{y}$. The next step is to twist the polarizer by an angle $\varphi$ and to make the second measurement in order to obtain $U_{\mathrm{PD} 1}^{\prime}$ and $U_{\mathrm{PD} 2}^{\prime}$ values. The twist angle $\varphi$ must be different from $90^{\circ}, 180^{\circ}, 270^{\circ}$. Drawing the line $t$ that is tangent to ellipse of polarisation one could determine the angle $\delta$ of polarisation state under investigation, where $\delta$ is the phase difference between two orthogonal, linearly polarized waves that come from decomposition of elliptically polarized wave. One can show that the angles $\delta, \alpha, v$ which determine the ellipse of polarization are [6]:

$$
\begin{aligned}
& \delta=\arccos \left(\frac{U_{\mathrm{PD} 1}^{\prime}-U_{\mathrm{PD} 1} \cos ^{2} \varphi-U_{\mathrm{PD} 2} \sin ^{2} \varphi}{\sqrt{U_{\mathrm{PD} 1} U_{\mathrm{PD} 2}} \sin 2 \varphi}\right), \\
& \alpha=0.5 \arctan \left[\frac{2\left(U_{\mathrm{PD} 1}^{\prime}-U_{\mathrm{PD} 1} \cos ^{2} \varphi-U_{\mathrm{PD} 2} \sin ^{2} \varphi\right)}{\left(U_{\mathrm{PD} 1}-U_{\mathrm{PD} 2}\right) \sin 2 \varphi}\right], \\
& |v|=0.5 \arcsin \left[\frac{2 \sqrt{U_{\mathrm{PD} 1} U_{\mathrm{PD} 2} \sin ^{2} 2 \varphi-\left(U_{1}^{\prime}-U_{1} \cos ^{2} \varphi-U_{2} \sin ^{2} \varphi\right)^{2}}}{\left(U_{\mathrm{PD} 1}+U_{\mathrm{PD} 2}\right)|\sin 2 \varphi|}\right] .
\end{aligned}
$$

In paper [6] the way of the angles $\delta, \alpha, v$ calculation for determination of the full ellipse of polarization are presented.

\section{Results of measurements}

The method worked out was applied to investigate the magnetooptic effects in step -index fibers, used in the system for magnetic field measurements. Various optical 


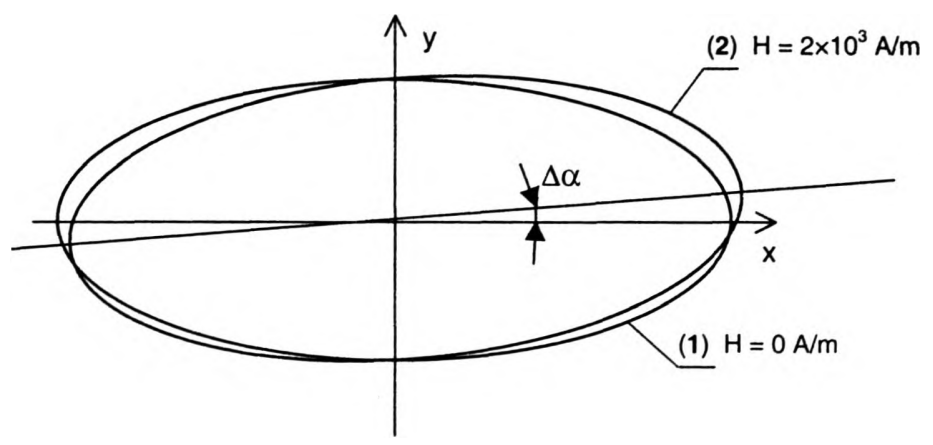

Fig. 3. Changes of the ellipse of the polarization state in fiber No. 1 due to the influence of the external magnetic field.

fibers were tested. We have been using the optical fibers manufactured in the Laboratory of Optical Fiber Technology at the University of Lublin, Poland. The results of investigation into the fibre properties are presented in Figs. 3, 4 and 5, the fibers being denoted by 1 and 2, (manufacturers symbols: SM960209 and Sm990112, respectively). They had the following parameters: core diameters $d_{\mathrm{cl}}=5 \mu \mathrm{m}$ and $d_{\mathrm{c} 2}=6 \mu \mathrm{m}$, diameter of claddings $d_{\mathrm{clad} 1,2} \approx 125 \mu \mathrm{m}$, and diameter of coatings $d_{\text {coat } 1.2} \approx 250 \mu \mathrm{m}$. The lengths of the fibers subjected to magnetic field were equal $l \approx 7 \mathrm{~m}$. The linearly polarized light was introduced at the fiber output. The optical power associated with orthogonal polarization planes, as a function of magnetic field intensity $H$, was measured at the fiber ends, which were some tens of meters long. The ellipses of the state of polarization, determined for fiber No. 1, using the method presented above is shown in Fig. 3 (for $H=0$ and $H=5 \times 10^{4} \mathrm{~A} / \mathrm{m}$ ).

Based on experimental results the values of angles $\alpha$ and $|v|$ were calculated. The calculated parameters were burdened with uncertainties that arose from uncertainties of voltage measurements and defining the angle $\varphi$. When the intensity of a magnetic

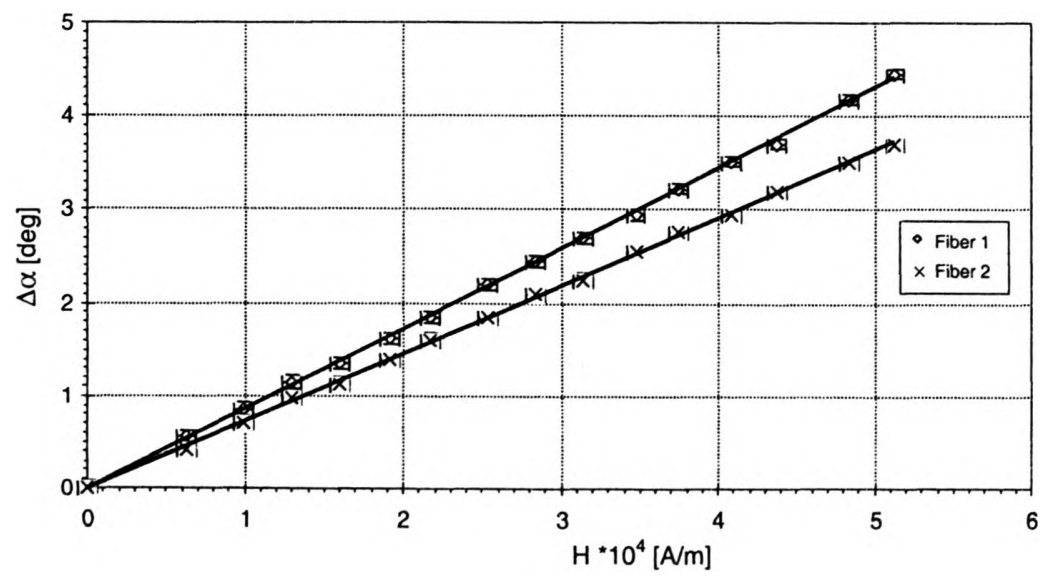

Fig. 4. Changes of the azimuth $\Delta \alpha$ of the polarization state as a function of magnetic field intensity. 


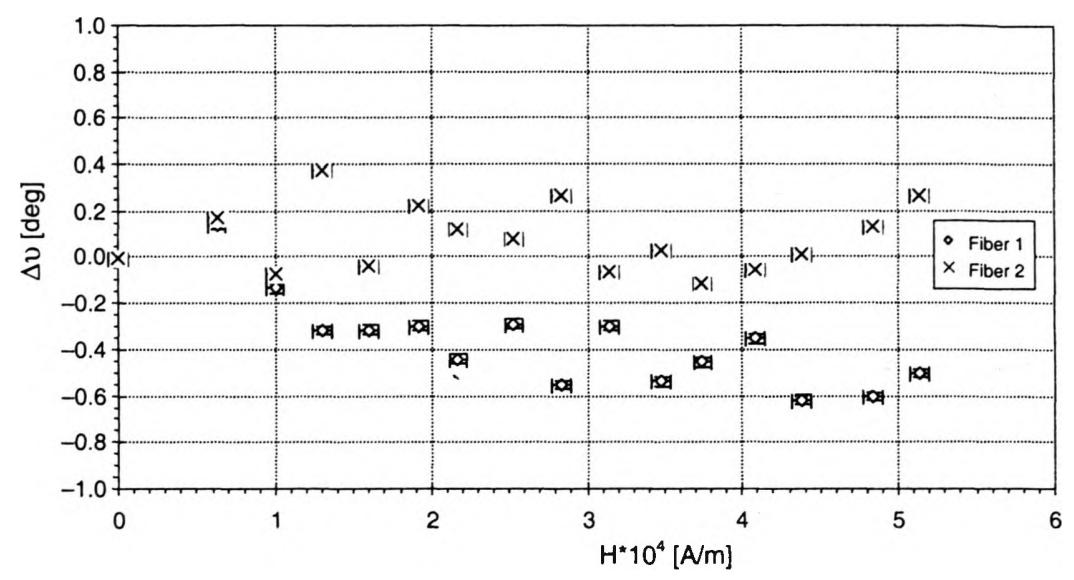

Fig. 5. Changes of the ellipticity of the polarization state in external magnetic field.

field is equal to zero, the azimuth is equal to zero, too. Therefore the values of angle $\alpha$ are equal to the twisting angle of polarization plane $\Gamma$. The dependence between $\Gamma$ and $H$ is shown in Figure 4.

In Figure 5, the changes of ellipticity induced by magnetic field changes are insignificant and of a rather accidental character.

The measurements show that the azimuth depends linearly on the magnetic field intensity. Therefore, the linear functions were fitted to the measurement points at $\Gamma(H) \equiv \alpha(H)$ plot. The fit was done using Gaussian iterative method. The fitting coefficients are: $a_{1}=(3.80 \pm 0.05) \mathrm{rad} \cdot \mathrm{m} / \mathrm{A}, a_{2}=(2.55 \pm 0.05) \mathrm{rad} \cdot \mathrm{m} / \mathrm{A}$.

The value of Verdet's constant was calculated based on the fitting coefficients. Since Verdet's constant is defined as $\alpha(H)=V l H$, then its value is given by the formula $V=a / l$. Placing the suitable values one obtains: $V_{1}=(0.40 \pm 0.01) \times 10^{-6} \mathrm{rad} / \mathrm{A}$, $V_{2}=(0.35 \pm 0.01) \times 10^{-6} \mathrm{rad} / \mathrm{A}$.

In work [6], the Verdet's constants determined for the other quartz fibers had similar values $\left(V \approx(0.30 \pm 0.01) \times 10^{-6} \mathrm{rad} / \mathrm{A}\right)$.

\section{Conclusions}

Very small and accidental changes of an angle of ellipticity $|v|$ for applied fibers suggest that the magnetic field does not change their ellipticity. For this reason one may assume that the magnetic field induces such changes of elliptical birefringence in the optical fibers tested that the angles $\alpha$ depend linearly on magnetic field intensity [6], [7].

It should be emphasized that quartz fibers are very sensitive to accidental changes of stress and small deformations. This feature causes that simple single-mode fiber may be in appropriate for application in this type of sensors.

For magnetic field sensors, new optical fibers based on magnetic glasses have been produced. The results of testing the new sensors will be published, elsewhere. 
Acknowledgments - The work was sponsored by the Polish State Committee for Scientific Research (KBN) within the Grant 8 T11B 04518.

\section{References}

[1] Jaroszewicz L.R., Coherence and Birefringence in Optical Fiber Interference, WAT Publishers, Warszawa 1996.

[2] Pustelny T., Tyszkiewicz C., Barczak K., Molecular and Quantum Acoustic 21 (2000), 219.

[3] Ibidem p. 207.

[4] KıEżun A., Detection System of Polarimeter, [In] VI Conference on Electron Technology ELTE, Wrocław 1997, pp. 114-117.

[5] Ratajczyk F., Birefringence and Optical Polarisation, Wrocław University of Technology Publishers, Wrocław 2000 (in Polish).

[6] Pustelny T., Barczak K., Tyszkiewicz C., Molecular and Quantum Acoustic 22 (2001), 203.

[7] Karasinski P., Rogozinski R., Molecular and Quantum Acoustic 21 (2000), 117.

[8] Grattan K.T.V., Meggitt B.T., Optical Fiber Sensor Technology, Chapmann \& Hall, London 1995.

[9] Iwamoto K., Appl. Opt. 29 (1990), 375.

[10] Hammer A., Proc. SPIE 932 (1996), 67.

Received November 15, 2002

in revised form January 20, 2003 